\title{
Seminormality and Upper Semicontinuity in Optimal Control ${ }^{1}$
}

\author{
LAMBerto Cesari ${ }^{2}$
}

\begin{abstract}
This paper concerns the concept of upper semicontinuity of variable sets, precisely the variant of Kuratowski's definition of upper semicontinuity that Cesari has denoted as property $(Q)$. This concept has been used by Cesari in most of his papers on existence theorems for optimal solutions, and later used by Olech, Lasota and Olech, Brunoysky, Baum, Suryanarayana, and Angell. First, criteria are given for property $(Q)$ in addition to those which had been already given previously. Then, it is shown that a slight restriction in the concept can be expressed in a form which is similar to Tonelli's concept of seminormality for free problems of the calculus of variations. Thus, the property $(Q)$ appears to be a generalization to Lagrange problems of control of the well-known concept of seminormality for free problems.
\end{abstract}

\section{Introduction}

In the present paper, we discuss properties of upper semicontinuity of variable, convex, closed sets in Euclidean spaces, taking into consideration the modification of Kuratowski's concept of upper semicontinuity (Ref. 1), which we denoted in Refs, $2-3$ as property $(Q)$. We have used this property in the proof of lower closure theorems in Lagrange and Mayer problems of optimal control. These theorems reduce to well-known lower semi-continuity statements for usual free problems of the calculus of variations. Lower closure theorems are used to prove existence theorems for Lagrange and Mayer problems of optimal control (Refs. 2-3). The same property $(Q)$ mentioned above was used again in recent studies by La Palm (Ref. 4), Lasota and Olech (Ref. 5), Olech (Refs. 6-7), Cesari, Nishiura, and La Palm (Ref. 8), and in recent papers by Cesari (Refs. 9-12) concerning existence

\footnotetext{
${ }^{1}$ Paper received December 19, 1969; in revised form, January 29, 1970. This research was partially supported by AFOSR Research Project No. 69-1662.

${ }^{2}$ Professor of Mathematics, University of Michigan, Ann Arbor, Michigan.
} 
theorems for Lagrange problems with multiple integrals and partial differential equations. In these papers, property $(Q)$ was requested for variable sets $\overline{Q(x)}$ of the form $\tilde{Q}(x)=\left[\left(z^{0}, z\right) \mid z^{0} \geqslant f_{0}(x, u), z=f(x, u), u \in U(x)\right] \subset E_{n+1}$, where $f_{0}$ and $f=\left(f_{1}, \ldots, f_{n}\right)$ are given continuous functions.

In the present paper, we give criteria (Section 3, 4, 7, 9) for property $(Q)$ of the sets $\widetilde{Q}(x)$ in addition to those already proved in Refs. 2-3 and those proved by Olech in Refs. 6-7. In particular, we show (Section 4 and 9) that a slight particularization of property $(Q)$ for these sets $\widetilde{Q}(x)$ can be expressed in a form which is similar to Tonelli's seminormality condition (Ref. 13) for free problems of the calculus of variations. Thus, property $(Q)$ of the sets $\widetilde{Q}(x)$ is shown here to represent a generalization for Lagrange problems of the well-known seminormality condition for free problems. In Sections $5,6,8$, we state a number of properties of convex, real-valued functions on a convex subset of $E_{n}$, related to the concept of seminormality, and we use these results in Section 9. In Section 7, we prove another criterion for property $(Q)$ of the sets $\mathscr{Q}(x)$ when $f$ is linear in $u$ and $f_{0}$ is convex and seminormal in $u$.

\section{Properties $(U)$ and $(Q)$ of Variable Sets}

Let $A$ be a given subset of the $x$-space $E_{r}$, for every $x \in A$ let $U(x)$ be a given subset of the $u$-space $E_{m}$, and let $M$ be the set of all $(x, u)$ with $x \in A$, $u \in U(x)$. Thus, $M$ is the graph of $U(x)$ in the space $E_{r} \times E_{m}$. For every $\bar{x} \in A$ and $\delta>0$, let $N_{\delta}(\bar{x})$ denote the set of all $x \in A$ at a distance $\leqslant \delta$ from $\bar{x}$. For every $\bar{x} \in A$ and $\delta>0$, let $U(\bar{x} ; \delta)$ denote the union of all $U(x)$ with $x \in N_{\delta}(\bar{x})$, or $U(\bar{x} ; \delta)=\left[u \in E_{m} \mid u \in U(x), x \in N_{\delta}(\bar{x})\right]$. We say that the sets $U(x)$ have property $(U)$ at a point $\bar{x} \in A$ if

$$
U(\bar{x})=\bigcap_{\delta} \operatorname{cl} U(\bar{x} ; \delta) .
$$

We say that the sets $U(x)$ have property $(Q)$ at $\bar{x} \in A$ if

$$
U(\bar{x})=\bigcap_{\delta} \mathrm{cl} \operatorname{co} U(\bar{x} ; \delta) .
$$

Here, $\mathrm{cl}$ and co denote the closure and the convex hull, respectively, of the sets under consideration. We say that the sets $U(x)$ have property $(U)[(Q)]$ in $A$ if this property holds at every point $\bar{x} \in A$. Property $(U)$ is Kuratowski's concept of uppersemicontinuity of sets (Ref. 1) and was used, for instance, by Choquet (Ref. 14) and Michael (Ref. 15). 
Note that in (1)-(2) the sign $C$ holds trivially, and thus the actual requirements can be written in the form

$$
U(\bar{x}) \supset \bigcap_{\delta} \operatorname{cl} U(\bar{x} ; \delta) \quad \text { or } \quad U(\bar{x}) \supset \bigcap_{\delta} \operatorname{cl} \operatorname{co} U(\bar{x} ; \delta),
$$

respectively. The following statements are easily proved:

(2.i) If $U(x)$ has property $(U)$ at $\bar{x}$, then $U(\bar{x})$ is closed.

(2.ii) If $U(x)$ has property $(Q)$ at $\bar{x}$, then $U(\bar{x})$ is closed and convex.

(2.iii) If $A$ is closed, then $U(x)$ has property $(U)$ in $A$ if, and only if, $M$ is closed.

A number of other statements concerning properties $(U)$ and $(Q)$ have been stated in Refs, 2-3 and will not be repeated here.

If $f(x, u)=\left(f_{1}, \ldots, f_{n}\right),(x, u) \in M$, is a given vector function, $f: M \rightarrow E_{n}$, we shall denote by $Q(x) \subset E_{n}$ the set $Q(x)=f(x, U(x))$, or

$$
Q(x)=\left[z=\left(z^{1}, \ldots, z^{n}\right) \mid z=f(x, u), u \in U(x)\right] .
$$

\section{The Sets $\mathscr{Q}(x)$ and a First Criterion for Property $(Q)$}

In Lagrange problems of optimal control and the calculus of variations, besides the vector function $f(x, u)=\left(f_{1}, \ldots, f_{n}\right)$, also a scalar function $f_{0}(x, u)$ is given, $f_{0}=M \rightarrow E_{1}$.

If $f(x, u)=\left(f_{0}, f\right)=\left(f_{0}, f_{1}, \ldots, f_{n}\right)$, then we may denote by $\tilde{Q}(x) \subset E_{n+1}$ the set $\tilde{f}(x, U(x))$, or $\widetilde{Q}(x)=\left[\left(z^{0}, z\right) \mid z^{0}=f_{0}(x, u), z=f(x, u), u \in U(x)\right]$. Also, we shall denote by $\bar{Q}(x)$ the set

$$
\widetilde{Q}(x)=\left[\left(z^{0}, z\right) \mid z^{0} \geqslant f_{0}(x, u), \quad z=f(x, u), \quad u \in U(x)\right] .
$$

We may say that $\widetilde{Q}(x)$ is the figurative and that $\tilde{Q}(x)$ is the set of points above the figurative. Note that, for every $x \in A$, the set $Q(x)$ is the projection on the $z$-space $E_{n}$ of the set $\widetilde{Q}(x) \subset E_{n+1}$. Thus, if $\widetilde{Q(x)}$ is convex, then certainly $Q(x)$ is also convex.

We shall say that a function $g(x, u)$ is of slower growth than $f_{0}(x, u)$ as $|u| \rightarrow \infty$ uniformly in some subset $A_{0}$ of $A$, provided given $\epsilon>0$ there is some $\bar{u}=\bar{u}\left(\epsilon, A_{0}\right) \geqslant 0$ such that $x \in A_{0}, u \in U(x),|u| \geqslant \bar{u}$ implies $|g(x, u)| \leqslant \epsilon f_{0}(x, u)$. 
(3.i) [A criterion for property $(Q)$ under a growth condition.] Given $A$ closed, $M$ closed, $f_{0}(x, u)$ and $f(x, u)=\left(f_{1}, \ldots, f_{n}\right)$ continuous on $M$, assume that 1 and $f$ are of slower growth than $f_{0}$ as $|u| \rightarrow+\infty$ uniformly on some neighborhood $A_{0}$ of a point $\bar{x} \in A$. If the set $\bar{Q}(\bar{x})$ is convex, then the sets $\Phi(x)$ satisfy property $(Q)$ at $\bar{x}$.

A proof of (3.i) has been given in Ref. 3 .

\section{A Second Criterion for Property $(Q)$}

Note that, if the sets $\mathscr{Q}(x)$ satisfy property $(Q)$ at a point $\bar{x} \in A$, then

$$
\tilde{Q}(\bar{x}) \supset \bigcap_{\delta} \mathrm{cl} \operatorname{co} \mathscr{Q}(\bar{x} ; \delta) \text {. }
$$

This means that, if a point $\left(z^{0}, z\right)$ belongs to the set $\bigcap_{\delta} \operatorname{cl} \operatorname{co} \widetilde{Q}(\tilde{x} ; \delta)$, then $\left(z^{0}, z\right) \in \Phi(\bar{x})=\left[\left(z^{0}, z\right) \mid z^{0} \geqslant f_{0}(\bar{x}, u), z=f(\bar{x}, u), u \in U(\bar{x})\right] ;$ hence,

$$
z \in Q(\bar{x})=[z=f(\bar{x}, u), \quad u \in U(\bar{x})] .
$$

In other words, the following property $(\alpha)$ is a necessary condition for the sets $\tilde{Q}(x)$ to have property $(Q)$ at $\vec{x}$ :

( $\alpha)$ If $\left(z^{0}, z\right) \in \bigcap_{\delta} \operatorname{cl} \operatorname{co} \bar{Q}(\bar{x} ; \delta)$, then $z \in Q(\bar{x})$.

For free problems of the calculus of variations, $n=m, f=u$, or $f_{i}=u^{i}$, $i=1, \ldots, n$, and $U(x)=E_{n}$. For these problems, the sets under consideration reduce to $Q(x)=E_{n}$ and

$$
\begin{array}{ll}
\widetilde{Q}(x)=\left[\left(z^{0}, u\right) \mid z^{0}=f_{0}(x, u),\right. & \left.u \in E_{n}\right] \subset E_{n+1}, \\
\widetilde{Q}(x)=\left[\left(z^{0}, u\right) \mid z^{0} \geqslant f_{0}(x, u),\right. & \left.u \in E_{n}\right] \subset E_{n+1} .
\end{array}
$$

Thus, property $(\alpha)$ is trivially satisfied for free problems.

We shall now introduce the following condition $(X)$ at a point $\bar{x} \in A$ :

$(X)$ For every $\bar{z} \in Q(\bar{x})$, there is at least one point $\bar{u} \in U(\bar{x})$ with $\bar{z}=f(\bar{x}, \bar{u})$ and the following property: given $\epsilon>0$, there are numbers $\delta>0$ and $r, b=\left(b_{1}, \ldots, b_{n}\right)$ real such that

$\left(X^{\prime}\right) \quad f_{0}(x, u) \geqslant r+\sum_{j} b_{j} f_{j}(x, u) \quad$ for all $\quad x \in N_{\delta}(\bar{x})$ and $u \in U(x)$,

$\left(X^{\prime \prime}\right) \quad f_{0}(\bar{x}, \bar{u}) \leqslant r+\sum_{j} b_{j} f_{j}(\bar{x}, \bar{u})+\epsilon$. 
For free problems (that is, $m=n, f=u, U=E_{n}$ ), the present property $(X)$ reduces to the following one concerning the function $f_{0}$ only:

$\left(X_{f}\right)$ For every $\bar{u}=\left(\bar{u}^{1}, \ldots, \bar{u}^{m}\right) \in E_{m}$ and $\epsilon>0$, there are numbers $\delta>0$ and $r, b=\left(b_{1}, \ldots, b_{m}\right)$ real such that

$\left(X_{f}^{\prime}\right) \quad f_{0}(x, u) \geqslant r+\sum_{j} b_{j} u^{j}$ for all $x \in N_{\delta}(\bar{x})$ and all $u=\left(u^{1}, \ldots, u^{m}\right) \in E_{m}$, $\left(X_{f}^{\prime \prime}\right) \quad f_{0}(\bar{x}, \bar{u}) \leqslant r+\sum_{j} b_{j} \bar{u}^{j}+\epsilon$.

As we shall see in Section 6 below, this condition $\left(X_{f}\right)$ is the well-known weak seminormality condition of the function $f_{0}$ at $(\bar{x}, \bar{u})$ for all $\bar{u} \in E_{m}$.

$(4, \mathrm{i}) \quad[$ A criterion for property $(Q)$ under conditions $(\alpha)$ and $(X)$.] If conditions $(\alpha)$ and $(X)$ hold at the point $\bar{x} \in A$, then $\widetilde{Q}(\bar{x})$ is closed and convex, and the sets $\widetilde{Q}(x)$ satisfy property $(Q)$ at the point $\bar{x}$.

Proof. To prove that the sets $\tilde{Q}(x)$ satisfy property $(Q)$ at $\bar{x}$ [and, hence, $\mathscr{Q}(\bar{x})$ is closed and convex], we have only to prove that, if $\tilde{z}=\left(\bar{z}^{0}, \bar{z}\right) \in \bigcap_{\delta} \mathrm{cl} \operatorname{co} \widetilde{Q}(\bar{x} ; \delta)$, then $\tilde{z}=\left(\bar{z}^{0}, \bar{z}\right) \in \widetilde{Q}(\bar{x})$. From condition $(\alpha)$, all we know is that $\bar{z} \in Q(\bar{x})$. Hence, there is some $\bar{u} \in U(\bar{x})$ such that $\bar{z}=f(\bar{x}, \bar{u})$ [hence, $(\bar{x}, \bar{u}) \in M]$, and statements $\left(X^{\prime}\right),\left(X^{\prime \prime}\right)$ hold for a suitable choice of $\bar{u}$.

For every $\delta>0$, we have $\tilde{z}=\left(\bar{z}^{0}, \bar{z}\right) \in \operatorname{cl} \operatorname{co} \delta(x ; \delta)$, and thus, for every $\delta>0$, there are points $\tilde{z}=\left(z^{0}, z\right) \in \operatorname{co} \tilde{Q}(\tilde{x} ; \delta)$ at a distance as small as we want from $\tilde{z}=\left(\bar{z}^{0}, \bar{z}\right)$. Thus, there is a sequence of numbers $\delta_{k}>0$ and of points $\tilde{z}_{k}=\left(z_{k}{ }^{0}, z_{k}\right) \in \operatorname{co} \widetilde{Q}\left(\bar{x} ; \delta_{k_{k}}\right)$ such that $\delta_{k} \rightarrow 0, \tilde{z}_{k} \rightarrow \tilde{z}$ as $k \rightarrow \infty$. In other words, for every integer $k$, there is a system of points $x_{k}^{\gamma} \in N_{\delta_{k}}(\bar{x})$, $\gamma=1, \ldots, \nu$, say $\nu=n+2$, corresponding points $\tilde{z}_{k}{ }^{\gamma}=\left(z_{k}^{0}, z_{k}^{\gamma}\right) \in \widetilde{Q}\left(x_{k}{ }^{\gamma}\right)$, points $u_{k}{ }^{\gamma} \in U\left(x_{k}^{\gamma}\right)$, and numbers $\lambda_{k}{ }^{\gamma} \geqslant 0, \gamma=1, \ldots, \nu$, such that

$$
\begin{gathered}
1=\sum \lambda_{k}{ }^{\gamma}, \quad z_{z_{k}}=\sum \lambda_{k}{ }^{\gamma} \tilde{z}_{k}{ }^{\gamma}, \quad z_{k}^{0}=\sum \lambda_{k}{ }^{\gamma} z_{k}^{0 \gamma}, \quad z_{k}=\sum \lambda_{k}{ }^{\gamma} z_{k}{ }^{\nu}, \\
z_{k}^{0 \gamma} \geqslant f_{0}\left(x_{k}{ }^{\gamma}, u_{k}{ }^{\gamma}\right), \quad z_{k}^{\gamma}=f\left(x_{k}{ }^{\gamma}, u_{k}{ }^{\gamma}\right),
\end{gathered}
$$

where $\gamma=1, \ldots, v$, where $\sum$ ranges over all $\gamma=1, \ldots, v$, and $z_{k}^{\gamma} \rightarrow \bar{x}, \tilde{s}_{k} \rightarrow \tilde{z}$, $z_{k}^{0} \rightarrow \bar{z}^{0}, z_{k} \rightarrow \bar{z}$ as $k \rightarrow \infty, \gamma=1, \ldots, \nu$.

By condition $\left(X^{\prime}\right)$, there is a neighborhood $N_{\delta}(\bar{x})$ of $\bar{x}$ in $A$ and numbers $r$, $b=\left(b_{1}, \ldots, b_{\nu}\right)$ real such that

$$
\begin{aligned}
& \bar{f}_{0}(x, u)=f_{0}(x, u)-r-b \cdot f(x, u) \geqslant 0 \quad \text { for all } \quad x \in N_{\delta}(\bar{x}) \text { and } \quad u \in U(x), \\
& \bar{f}_{0}(\bar{x}, \bar{u})=f_{0}(\bar{x}, \bar{u})-r-b \cdot f(\bar{x}, \bar{u}) \leqslant \epsilon .
\end{aligned}
$$


For $k$ sufficiently large, so that $\left|x_{k}{ }^{\gamma}-\bar{x}\right|<\delta, \gamma=1, \ldots, \nu$, we have now, from (3)-(4),

$$
\begin{aligned}
z_{k}^{0} & =\sum \lambda_{k}^{\gamma} z_{k}^{0 \gamma} \geqslant \sum \lambda_{k}^{\gamma} f_{0}\left(x_{k}^{\gamma}, u_{k}^{\gamma}\right) \geqslant \sum \lambda_{k}^{\gamma}\left[r+b \cdot f\left(x_{k}^{\gamma}, u_{k i}^{\gamma}\right)\right] \\
& =\sum \lambda_{k}^{\gamma}\left[r+b \cdot z_{k}^{\gamma}\right]=r+b \cdot \sum \lambda_{k}^{\gamma} z_{k}^{\gamma}=r+b \cdot z_{k} .
\end{aligned}
$$

As $k \rightarrow \infty$, we obtain $\bar{z}^{0} \geqslant r+b \cdot \bar{z}$; hence, from (5),

$$
\bar{z}^{0} \geqslant r+b \cdot \bar{z}=r+b \cdot f(\bar{x}, \bar{u})=f_{0}(\bar{x}, \bar{u})-f_{0}(\bar{x}, \bar{u}) \geqslant f_{0}(\bar{x}, \bar{u})-\epsilon .
$$

Here, $\epsilon>0$ is arbitrary; hence, $\bar{z}^{0} \geqslant f_{0}(\bar{x}, \vec{u})$, while $\bar{z}=f(\bar{x}, \bar{u})$. This shows that $\tilde{z}=\left(\bar{z}^{0}, \bar{z}\right) \in \tilde{Q}(\bar{x})$. We have proved that the sets $\widetilde{Q}(x)$ satisfy property $(Q)$ at the point $\bar{x} \in A$. Statement (4.i) is thereby proved.

\section{Some Properties of Convex Functions}

If $U$ is a given subset of $E_{n}$ and $F(u), u \in U$, a real-valued function, then $F(u)$ is said to be convex in $u$ provided $U$ is convex, and $u_{1}, u_{2} \in U, 0 \leqslant \alpha \leqslant 1$, implies $F\left(\alpha u_{1}+(1-\alpha) u_{2}\right) \leqslant \alpha F\left(u_{1}\right)+(1-\alpha) F\left(u_{2}\right)$. The following statements are well known:

(5.i) If $U$ is a convex subset of $E_{n}$ and $F(u), u \in U$, a given real-valued function, then $F(u)$ is convex if, and only if, $u_{j} \in U, \lambda_{j} \geqslant 0, j=1, \ldots, \nu$, $\nu$ finite, $\lambda_{1}+\cdots+\lambda_{\nu}=1, u_{0}=\sum_{j=1}^{\nu} \lambda_{j} u_{j}$, implies $F\left(u_{0}\right) \leqslant \sum_{j=1}^{\nu} \lambda_{j} F\left(u_{j}\right)$.

(5.ii) If $U$ is a convex subset of $E_{n}$ and $F(u), u \in U$, a given real-valued function, then $F(u)$ is convex if, and only if, the set

$$
\tilde{Q}=[(z, u) \mid z \geqslant F(u), u \in U] \subset E_{n+1}
$$

is convex.

A linear scalar function

$$
z(u)=r+b_{1} u^{1}+\cdots+b_{n} u^{n}=r+b \cdot u, \quad u \in E_{n},
$$

$r, b_{1}, \ldots, b_{n}$ real, is said to be a supporting plane of $F(u), u \in U$, at a point $\bar{u} \in U$, provided $F(\bar{u})=z(\bar{u})$ and $F(u) \geqslant z(u)$ for all $u \in U$.

(5.iii) If $U$ is a convex subset of $E_{n}$ and $F(u), u \in U$, a given real-valued convex function, then $F(u)$ has a supporting plane at every interior point $\bar{u}$ of $U$. 
Proof. We know already that the set $\bar{Q}=[(z, u) \mid z \geqslant F(u), u \in U] \subset E_{n+1}$ is convex, and hence there exists some supporting hyperplane $V$ at $(\bar{z}, \bar{u})$, $\bar{z}=F(\bar{u})$. If $V=\left[(z, u) \mid p^{0} z+p \cdot u-c \geqslant 0\right], p^{0}, p=\left(p^{1}, \ldots, p^{n}\right), c$ real, then $p^{0} \bar{z}+p \cdot \bar{u}-c=0$ and $p^{0} z+p \cdot u-c \geqslant 0$ for all $u \in U$ and $z \geqslant F(u)$. Let us prove that $p^{0} \neq 0$. Indeed, if $p^{0}=0$, then we have $p \cdot \bar{u}-c=0$, $p \cdot u-c \geqslant 0$ for all $u \in U$. If $u_{1} \in E_{n}$ is a point where $p \cdot u_{1}-c>0$, and $\epsilon$ real, then for $u=u(\epsilon)=\epsilon u_{1}+(1-\epsilon) \bar{u}$ we have $p \cdot u(\epsilon)-c>0$ for all $\epsilon>0$, and $p u(\epsilon)-c<0$ for all $\epsilon<0$, with $u(\epsilon) \rightarrow \bar{u}$ as $\epsilon \rightarrow 0$. Since $\bar{u} \in$ int $U$, then both $u(\epsilon), u(-\epsilon)$ belong to $U$ for $\epsilon>0$ sufficiently small, and $p u(-\epsilon)-c<0$, a contradiction. We have proved that $p_{0} \neq 0$. Actually, we must have $p_{0}>0$, since $p^{0} z+p \cdot \bar{u}-c \geqslant 0$ for all $z \geqslant F(\bar{u})$. Finally, if we take $z(u)=(-p \cdot u+c) / p^{0}$, then $z(\bar{u})=F(\bar{u})$ and $F(u) \geqslant z(u)$ for all $u \in U$.

Given a set $U$, we denote as usual by int $U$ the subset of its interior points.

If $U$ has no interior points, that is, int $U=\varnothing$, statement (5.iii) has the following implication. First, let us denote by $R$ the hyperspace of $E_{n}$ of minimum dimension $r$ containing $U$. Then, $U \subset R \subset E_{n}, 0 \leqslant r \leqslant n$. If $U$ is reduced to a single point, then $R=U$ and $r=0$. Otherwise, $1 \leqslant r \leqslant n$, and we denote by $R$ int $U$ the certainly nonempty set of points of $U$ which are interior to $U$ with respect to $R$. Thus, int $U \subset R$ int $U \subset U \subset R \subset E_{n}$. Statement (5.iii) now has the following corollary:

(5.iv) Under the same hypotheses as in (5.iii), $F(u)$ has a supporting plane at every point $\bar{u} \in R$ int $U$.

The following statement also is relevant:

(5.v) Under the same hypotheses as in (5.iii), $F(u)$ is continuous at every point $\bar{u} \in R$ int $U$.

Proof. We may well assume that $U$ is not a single point, that $1 \leqslant r \leqslant n$, and $\operatorname{Rint} U \neq \varnothing$. Let $\bar{u}$ be any point $\bar{u} \in \operatorname{Rint} U$, and let $z=c+p \cdot u$ be the supporting plane at $\bar{u}$, so that $F(\bar{u})=c+p \cdot \bar{u}$. Assume, if possible, that for some $\sigma>0$ and sequence of points $u_{k} \in \operatorname{Rint} U, u_{k} \rightarrow \bar{u}$ as $k \rightarrow \infty$, we have $F\left(u_{k}\right)-F(\bar{u}) \leqslant-\sigma$ for all $k$. Then, $F\left(u_{k}\right) \geqslant c+p \cdot u_{k}$, and hence $-\sigma \geqslant F\left(u_{k}\right)-F(\bar{u}) \geqslant p \cdot\left(u_{k}-\bar{u}\right)$. As $k \rightarrow \infty$, we have $-\sigma \geqslant 0$, a contradiction. Assume now, if possible, that for some $\sigma>0$ and sequence of points $u_{k} \in R$ int $U, u_{k} \rightarrow \bar{u}$ as $k \rightarrow \infty$, we have $F\left(u_{k}\right)-F(\bar{u}) \geqslant \sigma$ for all $k$. Then, we can choose $r$ points $v_{j} \in U, j=1, \ldots, r$, independent in $R$, such that $\left|v_{j}-u\right|=\delta>0, j=1, \ldots, r$, and $\bar{u}=\sum_{j}(1 / r) v_{j}$. Since $u_{k} \rightarrow \bar{u}$, we have 
$u_{k}=\sum_{j} \lambda_{j k} v_{j}$ with $\lambda_{j k} \rightarrow 1 / r$ as $k \rightarrow \infty, j=1, \ldots, r$. If $\lambda_{k}=\min \left[\lambda_{j k}, j=1, \ldots, r\right]$, then $0 \leqslant \lambda_{k} \leqslant 1 / r, u_{k}=\sum_{j}\left(\lambda_{j k}-\lambda_{k}\right) v_{j}+\left(\lambda_{k} r\right) \bar{u}$, and hence

$$
F\left(u_{k}\right) \leqslant \sum_{j}\left(\lambda_{j k}-\lambda_{k}\right) F\left(v_{k}\right)+\left(\lambda_{k} r\right) F(\bar{u})
$$

where $\lambda_{j k}-\lambda_{k} \rightarrow 0, \lambda_{k} r \rightarrow 1$. For all $k$ sufficiently large, we have then $F\left(u_{l_{c}}\right) \subseteq F(\bar{u})+\sigma / 2$, a contradiction. This proves that $F$ is continuous at every point $\bar{u} \in R$ int $U$.

(5.vi) Under the same hypotheses as in (5.iii), $F(u)$ is bounded below on every bounded part $K$ of $U$.

Proof. Indeed, if $K$ contains more than one point, then $K$ contains some point $\bar{z} \in \operatorname{Rint} U$, and, if $z(u)=c+p \cdot u$ is a supporting plane at $\bar{u}$, then $F(u) \geqslant c+p \cdot u$ for all $u \in K C U$, and $c+p \cdot u$ has a finite lower bound on $K$.

(5.vii) Under the same hypotheses as in (5.iii), $F(u)$ is upper semicontinuous at every point $\bar{u} \in U-R$ int $U$ along any segment $s$ issued from $\bar{u}$ and contained in $U$.

Proof. Let $s$ be the segment $s=\bar{u} u_{0}, s \subset U$. Assume, if possible, that there is a sequence of points $u_{k} \in s \subset U, u_{k} \rightarrow \bar{u}$ as $k \rightarrow \infty$, with $F\left(u_{k}\right)-F(\bar{u}) \geqslant \sigma$ for all $k$. Then, all points interior to the segment $s$ are certainly points of $\operatorname{Rint} U$, say $u=(1-\alpha) \bar{u}+\alpha u_{0}, 0<\alpha<1$, and since $F(u) \leqslant(1-\alpha) F(\bar{u})+\alpha F\left(u_{0}\right)$, we see that $F$ is bounded above on $s$. Since $h_{k}=u_{k}-\bar{u} \rightarrow 0$ as $k \rightarrow \infty$, there is a sequence of numbers $\beta_{k}>1$ with $\beta_{k} \rightarrow \infty, \beta_{k} h_{k} \rightarrow 0$ as $k \rightarrow \infty$. Hence, the points $u_{k}^{\prime}=\bar{u}+\beta_{k}\left(u_{k}-\bar{u}\right)$, $k=1,2, \ldots$, are on the straight line from $\bar{u}$ containing $s$, and $u_{k}{ }^{\prime} \rightarrow \bar{u}$ as $k \rightarrow \infty$. Thus, $u_{k}{ }^{\prime} \in s, u_{k}{ }^{\prime} \in \operatorname{Rint} U$ for all $k$ sufficiently large, and the following relations hold:

$$
\begin{aligned}
u_{k} & =\beta_{k}^{-1} u_{k}^{\prime}+\beta_{k}^{-1}\left(\beta_{k}-1\right) \bar{u}, \\
F\left(u_{k}\right) & \leqslant \beta_{k}^{-1} F\left(u_{k}\right)+\beta_{k}^{-1}\left(\beta_{k}-1\right) F(\bar{u}), \\
F\left(u_{k}\right) & \geqslant \beta_{k} F\left(u_{k}\right)-\left(\beta_{\tilde{k}}-1\right) F(\bar{u}) \geqslant F(\bar{u})+\beta_{k} \sigma .
\end{aligned}
$$

Hence, $F\left(u_{k}{ }^{\prime}\right) \rightarrow+\infty$ as $k \rightarrow+\infty$, a contradiction since $F$ is bounded above on $s$. We have proved that $F$ is upper semicontinuous at $\bar{u}$ along $s$.

A function $F(u), u \in U$, convex on a convex set $U$, may not be continuous at the points of $U-R$ int $U$, as the following example shows. Take $U=[u \mid 0 \leqslant u \leqslant 1]$, and $F(u)=0$ for $0<u<1, F(u)=1$ for $u=0$ and $u=1$. 
(5.viii) If $U$ is a convex subset of $E_{n}$, if $F(u), u \in U$, is a given realvalued convex function on $U$, and the set $\widetilde{Q}=\left[\left(z^{0}, u\right) \mid z^{0} \geqslant F(u), u \in U\right]$ is convex and closed, then the function $F(u)$ is lower semicontinuous at every point $\bar{u} \in U-R$ int $U$, and even continuous on each segment $s$ issued from $\bar{u}$ and contained in $U$.

Proof. Assume, if possible, that there is a number $\sigma>0$ and points $\bar{u}$, $u_{k}, k=1,2, \ldots$, with $\bar{u} \in U-\operatorname{Rint} U, u_{k} \in U, F\left(u_{k}\right)<F(\bar{u})-\sigma$ for all $k$. Take $\bar{z}^{0}=F(\bar{u})$, and note that all points $\left(\bar{z}^{0}-\sigma, u_{k}\right)$ are in $\tilde{Q}$. Then, as $k \rightarrow \infty$, we see that $\left(z^{0}-\sigma, \bar{u}\right)$ is in the closed set $\bar{Q}$, a contradiction, since $(z, \bar{u}) \in \widetilde{Q}$ if, and only if, $z \geqslant \bar{z}^{0}=F(\bar{u})$. The last part of the statement is a consequence of (5.vii).

A function $F(u), u \in U$, convex on a convex set $U$, may not be continuous at the points of $U$-Rint $U$, even if the set $\bar{Q}$ is closed, as the following example shows. Take $U=\left[(u, v) \mid 0 \leqslant u \leqslant 1,0 \leqslant v \leqslant \sqrt{ }\left[1-(1-u)^{2}\right]\right.$, $F(u, v)=v$ if $0 \leqslant u \leqslant 1,0 \leqslant v \leqslant u, F(u, v)=(2 u)^{-1}\left(u^{2}+v^{2}\right)$ if $0<u<1$, $u \leqslant v \leqslant \sqrt{ }\left[1-(1-u)^{2}\right]$. Obviously, $U$ is convex, $F$ is convex in $(u, v)$, but $F$ is not continuous at $(0,0)$, since $F(0,0)=0, F\left(u, \sqrt{ }\left[1-(1-u)^{2}\right]\right)=1$ for all $0<u<1$.

Given a convex set $U \subset E_{n}$ and a scalar function $F(u), u \in U$, we say that $F(u)$ is convex at the point $\bar{u} \in U$ provided $F(\bar{u}) \leqslant \sum_{j=1}^{v} \lambda_{j} F\left(u_{j}\right)$ for any convex combination $\bar{u}=\sum_{j=1}^{v} \lambda_{j} u_{j}$ of points $u_{j} \in U$.

(5.ix) If $U$ is a convex subset of $E_{n}$ and $F(u), u \in U$, a given real-valued function, then $F(u)$ is convex at an interior point $\bar{u}$ of $U$ if, and only if, $F(u)$ has a supporting plane at $\bar{u}$.

A proof of this statement can be found in Turner (Ref. 16). We repeat here the proof for the convenience of the reader.

Proof. Suppose $F$ convex at the point $\bar{u} \in$ int $U$. Then, the smallest convex set co $\check{Q}$ containing $\widetilde{Q}=[(z, u) \mid z \geqslant F(u), u \in U] \subset E_{n+1}$ is the set of all points $(\approx, u)=\sum_{j=1}^{v} \lambda_{j}\left(z_{j}, u_{j}\right)$ with $\left(z_{j}, u_{j}\right) \in \bar{Q}, \lambda_{j} \geqslant 0, \lambda_{1}+\cdots+\lambda_{\nu}=1$, $\nu$ finite. Now, $(z, \bar{u}) \notin$ co $\mathscr{Q}$ if $z<F(\bar{u})$ since, for every convex combination $(z, u)=\sum_{j=1}^{\nu} \lambda_{j}\left(z_{j}, u_{j}\right)$ with $u=\bar{u}, \bar{u}=\sum_{j}^{\nu} \lambda_{j} u_{j}$, we have $z=\sum_{j} \lambda_{j} z_{j} \geqslant$ $\sum_{j} \lambda_{j} F\left(u_{j}\right) \geqslant F(\bar{u})$. Hence, $z>z_{0}$, and therefore $(F(\bar{u}), \bar{u})$ is a boundary point of co $\mathscr{Q}$. Then, there is a hyperplane $V=\left[(z, u) \mid p_{0} z+p \cdot u-c=0\right] \subset E_{n+1}$ such that $p_{0} F(\bar{u})+p \cdot \bar{u}-c=0$ and $p_{0} z+p \cdot u-c \geqslant 0$ for all $(z, u) \in \operatorname{co} \bar{Q}$.

For every convex combination $\bar{u}=\sum \lambda_{j} u_{j}$ and numbers $z_{j} \geqslant F\left(u_{j}\right)$, we have $\left(z_{j}, u_{j}\right) \in \operatorname{co} Q$, and $p_{0} z_{j}+p \cdot u_{j}-c \geqslant 0$. Therefore, $p_{0}\left[\sum_{j} \lambda_{j} z_{j}\right]+p \cdot \bar{u}-c \geqslant 0$, $\dot{p}_{0} F(\bar{u})+p \cdot \bar{u}-c=0$, and $p_{0}\left[\sum \lambda_{j} z_{j}-F(\bar{u})\right] \geqslant 0$. Since this is true for 
arbitrary large $z_{j}$ and $\lambda_{j} \geqslant 0$, we conclude that $p_{0} \geqslant 0$. But $p_{0}=0$ implies $p \cdot u-c \geqslant 0$ for all $u \in U$, which is impossible, as in the previous proof. Thus, $p_{0}>0$, and the hyperplane $V$ can be written now in the form $z=b \cdot u+r$, with $b=-p / p_{0}, r=-c / p_{0}$, and $z \geqslant b \cdot u+r$ for all $(z, u) \in \operatorname{co} \tilde{Q}, F(\bar{u})=b \cdot \bar{u}+r$. Thus, $z(u)=b \cdot u+r$ is a supporting plane for $F(u)$ at $u=\bar{u}$.

Conversely, if $F(u)$ has a supporting plane $z(u)=b \cdot u+r$ at $\bar{u} \in U$, then, for every convex combination $\bar{u}=\sum_{j} \lambda_{j} u_{j}$ of points $u_{j} \in U$, we have $\sum_{j} \lambda_{j} F\left(u_{j}\right) \geqslant \sum_{j} \lambda_{j} z\left(u_{j}\right)=\sum_{j} \lambda_{j}\left[b \cdot u_{j}+r\right]=b \cdot \bar{u}+r=F(\bar{u})$, and $F(u)$ is convex at $\bar{u}$.

The following statement $(5 \times \mathrm{x})$ concerns the case where $U=E_{\imath z}, F(u)$ is convex in $u$ in $E_{n}$, and $(5 . x)$ gives a characterization of those $F$ that are linear on no straight line of $E_{n}$.

(5.x) If $F(u), u \in E_{n}$, is convex in $u$, then there are no points $u_{0}, u_{1} \in E_{n}$ with $u_{1} \neq 0$ such that

$$
F\left(u_{0}\right)=2^{-1}\left[F\left(u_{0}+\lambda u_{1}\right)+F\left(u_{0}-\lambda u_{1}\right)\right] \text { for all real } \lambda,
$$

if, and only if, there is a linear function $w(u)=\bar{r}+\bar{b} \cdot u, u \in E_{n}, \bar{r}$, $\bar{b}=\left(\bar{b}_{1}, \ldots, \bar{b}_{n}\right)$ real, such that $F(u) \geqslant w(u)$ for all $u \in E_{n}$, and $F(u)-w(u) \rightarrow+\infty$.

This statement was essentially proved by Tonelli (Ref. 13) under smoothness conditions on $F$. The proof below, based only on continuity and convexity properties, can be found in Turner (Ref. 16) and is repeated here for the convenience of the reader.

Proof. (a) Let us prove the sufficiency. Assume, if possible, that such a linear function $w(u)$ as above exists and also that (6) holds for some $u_{0}, u_{1} \in E_{n}, u_{1} \neq 0$. Let $\approx(u)=r+b \cdot u, u \in E_{n}, r, b=\left(b_{1}, \ldots, b_{n}\right)$ real, be a supporting plane of $F(u)$ at $u_{0}$. Then, $F(u) \geqslant \approx(u)$ for all $u \in E_{n}$, and

$$
\begin{gathered}
F\left(u_{0}+\lambda u_{1}\right) \geqslant r+b \cdot\left(u_{0}+\lambda u_{1}\right), \\
F\left(u_{0}-\lambda u_{1}\right) \geqslant r+b \cdot\left(u_{0}-\lambda u_{1}\right), \\
F\left(u_{0}\right)=r+b \cdot u_{0} .
\end{gathered}
$$

By difference, we have

$$
\begin{aligned}
& F\left(u_{0}+\lambda u_{1}\right)-F\left(u_{0}\right) \geqslant b \cdot\left(\lambda u_{1}\right), \\
& F\left(u_{0}-\lambda u_{1}\right)-F\left(u_{0}\right) \geqslant b \cdot\left(-\lambda u_{1}\right),
\end{aligned}
$$


and, by using (6), also

$$
\begin{aligned}
& 2^{-1}\left[F\left(u_{0}+\lambda u_{1}\right)-F\left(u_{0}-\lambda u_{1}\right)\right] \geqslant b \cdot\left(\lambda u_{1}\right), \\
& 2^{-1}\left[F\left(u_{0}-\lambda u_{1}\right)-F\left(u_{0}+\lambda u_{1}\right)\right] \geqslant b \cdot\left(-\lambda u_{1}\right) .
\end{aligned}
$$

Since the sum of these relations is $0=0$, we conclude that the equality sign holds in both; hence,

$$
\begin{aligned}
& F\left(u_{0}+\lambda u_{1}\right)-F\left(u_{0}\right)=2^{-1}\left[F\left(u_{0}+\lambda u_{1}\right)-F\left(u_{0}-\lambda u_{1}\right)\right]=b \cdot\left(\lambda u_{1}\right), \\
& F\left(u_{0}-\lambda u_{1}\right)-F\left(u_{0}\right)=2^{-1}\left[F\left(u_{0}-\lambda u_{1}\right)-F\left(u_{0}+\lambda u_{1}\right)\right]=b \cdot\left(-\lambda u_{1}\right),
\end{aligned}
$$

and, finally,

$$
F\left(u_{0}+\lambda u_{1}\right)=F\left(u_{0}\right)+b \cdot\left(\lambda u_{1}\right)=r+b \cdot\left(u_{0}+\lambda u_{1}\right) .
$$

From $F(u) \geqslant w(u)$, we deduce now that

$$
F\left(u_{0}+\lambda u_{1}\right)=r+b \cdot\left(u_{0}+\lambda u_{1}\right) \geqslant \bar{r}+\bar{b} \cdot\left(u_{0}+\lambda u_{1}\right),
$$

and, hence,

$$
r-\bar{r}+(b-\bar{b}) \cdot u_{0} \geqslant \lambda(\bar{b}-b) \cdot u_{1}
$$

for all $\lambda$ real. Since the first member is a constant, we must have $(\bar{b}-b) \cdot u_{1}=0$, and then

$$
\begin{aligned}
F\left(u_{0}+\lambda u_{1}\right)-w\left(u_{0}+\lambda u_{1}\right) & =r+b \cdot\left(u_{0}+\lambda u_{1}\right)-\bar{r}-\bar{b} \cdot\left(u_{0}+\lambda u_{1}\right) \\
& =r-\bar{r}+(b-b) \cdot u_{0},
\end{aligned}
$$

where the last member is a constant. This contradicts that $F(u)-w(u) \rightarrow \infty$ as $|u| \rightarrow+\infty$. We have proved the sufficiency of the condition.

(b) Let us prove the necessity. First, assume that $F(u) \geqslant 0$ for all $u \in E_{n}$, with $F(0)=0$. Let $T$ be the set of all real vectors $b=\left(b_{1}, \ldots, b_{n}\right)$ for which there is some real number $r$ such that $F(u) \geqslant r+b \cdot u$ for all $u \in E_{n}$. If $b_{1}, b_{2} \in T$ and $r_{1}, r_{2}$ are the corresponding numbers, then, for $0 \leqslant \alpha \leqslant 1$,

$$
\begin{aligned}
F(u) & -\left[\alpha r_{1}+(1-\alpha) r_{2}-\left(\alpha b_{1}+(1-\alpha) b_{2}\right) \cdot u\right] \\
& =\alpha\left[F(u)-\left(r_{1}+b_{1} \cdot u\right)\right]+(1-\alpha)\left[F(u)-\left(r_{2}+b_{2} \cdot u\right)\right] \geqslant 0
\end{aligned}
$$

for all $u \in E_{n}$. Hence, $\alpha b_{1}+(1-\alpha) b_{2} \in T$, and $T$ is convex. Moreover, $T$ contains the origin since $F(u) \geqslant 0$ for all $u \in E_{n}$. 
Let us prove that $T$ is not contained in any $(n-1)$-dimensional subspace of $E_{n}$. If it were, there would be a unit vector $e$ such that $e \cdot b=0$ for all $b \in T$. Since $F(\lambda e)+F(-\lambda e)>0$ for some $\lambda \neq 0$, then either $F(\lambda e)>0$ or $F(-\lambda e)>0$. Suppose $F(\lambda e)>0$, to be concrete. Let $z(u)=F(\lambda e)+b \cdot(u-\lambda e)$ be a supporting plane for $F(u)$ at the point $\lambda e$. This supporting plane exists by force of (5.iii). Then, $F(u) \geqslant z(u)$ for all $u$, so $b \in T, e \cdot b=0$, and $z(\gamma e)=F(\lambda e)+b \cdot(\gamma e-\lambda e)=F(\lambda e)>0$ for all $\gamma$ real. Thus, in the directions $t e$, the function $z(u)$ is constant and positive. But $z(0) \leqslant F(0)=0$, a contradiction. Thus, $T$ is $n$-dimensional.

We know that a convex set in $E_{n}$ contained in no $(n-1)$-dimensional manifold has an interior point. Therefore, let $\bar{b}, \epsilon>0$, be such that $\bar{b} \in T$ and $|b-\bar{b}| \leqslant \epsilon$ implies $b \in T$. Let $\bar{r}$ be a constant such that $F(u) \geqslant w(u)=$ $\bar{r}+\bar{b} \cdot u$ for all $u \in E_{n}$. Suppose that $\lim \inf [F(u)-w(u)] \neq+\infty$, where lim inf is taken as $|u| \rightarrow+\infty$. Then, there is a constant $a>0$ and a sequence $\left[u_{k}\right]$ such that $\left|u_{k}\right| \rightarrow+\infty, F\left(u_{k}\right)-v\left(u_{k}\right)<a$ for all $k$. Without loss of generality, we can assume that $u_{k} /\left|u_{k}\right|$ converges to a unit vector $\ddot{u}$ as $k \rightarrow \infty$. Then, $\bar{b}+\epsilon \bar{u} \in T$, and there is a constant $r_{1}$ such that $\approx(u)=r_{1}+(b+\epsilon \bar{u}) \cdot u \leqslant F(u)$ for all $u$. Thus,

$$
\begin{aligned}
F\left(u_{k}\right)-w\left(u_{k}\right) & \geqslant r_{\mathbf{1}}+(\bar{b}+\epsilon \bar{u}) \cdot u_{k}-\bar{r}-\bar{b} \cdot u_{k} \\
& =r_{\mathbf{1}}-\overline{\boldsymbol{r}}+\epsilon \bar{u} \cdot u_{k} \\
& =r_{\mathbf{1}}-\bar{r}+\epsilon\left|u_{k}\right| \bar{u} \cdot\left(u_{k}|| u_{\bar{k}} \mid\right) \rightarrow+\infty
\end{aligned}
$$

as $k \rightarrow \infty$, a contradiction. Thus, $F(u)-w(u) \rightarrow+\infty$ as $|u| \rightarrow+\infty$.

We have proved the statement for functions $F$ with $F(u) \geqslant 0$ and $F(0)=0$. For an arbitrary $F(u)$, let $z(u)=F(0)+b_{1} \cdot u$ be a supporting plane for $F(u)$ at the origin. Let $G(u)=F(u)-z(u)$. Then, $G(u) \geqslant 0$ for all $u \in E_{n}$ and $G(0)=0$. Thus, $G$ satisfies the hypotheses assumed at the beginning, and there exists $w_{2}(u)=r_{2}+b_{2} \cdot u$ such that $G(u) \geqslant w_{2}(u)$ for all $u$ and $G(u)-w_{2}(u) \rightarrow+\infty$. Let $w(u)=z(u)+w_{2}(u)$. Then, $F(u)-w(u)=$ $G(u)-w_{2}(u)>0$ for all $u \in E_{n}$, and $\lim [F(u)-w(u)]=\lim \left[G(u)-w_{2}(u)\right]=+\infty$, where both limits are taken as $|u| \rightarrow+\infty$. Statement (5.x) is thereby proved.

\section{Seminormality of Convex Functions}

As usual, let $A$ be a closed subset of the $x$-space and $f_{0}(x, u)$ a given scalar function continuous on $A \times E_{n}$.

The function $f_{0}(x, u)$ is said to be weakly seminormal in $u$ at the point 
$(\bar{x}, \bar{u}) \in A \times E_{m}$ provided, given $\epsilon>0$, there are numbers $\delta>0$ and $r$, $b=\left(b_{1}, \ldots, b_{n}\right)$ real such that

$$
\begin{array}{ll}
\left(X_{f}^{\prime}\right) & f_{0}(x, u) \geqslant r+b \cdot u \quad \text { for all } \quad x \in N_{\delta}(\bar{x}), \quad u \in E_{m}, \\
\left(X_{f}^{\prime \prime}\right) & f_{0}(\bar{x}, \bar{u}) \leqslant r+b \cdot \bar{u}+\epsilon .
\end{array}
$$

The function $f_{0}(x, u)$ is said to be weakly seminormal in $u$ at the point $\bar{x} \in A$ if it has the just-mentioned property at $(\bar{x}, \bar{u}) \in A \times E_{m}$ for every $\bar{u} \in E_{m}$.

The function $f_{0}(x, u)$ is said to be seminormal in $u$ at the point $(\bar{x}, \bar{u}) \in A \times E_{m}$ provided, given $\epsilon>0$, there are numbers $\delta>0, \nu>0$, and $r, b=\left(b_{1}, \ldots, b_{n}\right)$ real such that

$\left(\mathrm{SN}^{\prime}\right) \quad f_{0}(x, u) \geqslant r+b \cdot u+\nu|u-\bar{u}| \quad$ for all $\quad x \in N_{\delta}(\bar{x}), \quad u \in E_{m}$,

$\left(\mathrm{SN}^{\prime \prime}\right) \quad f_{0}(\bar{x}, \bar{u}) \leqslant r+b \cdot \bar{u}+\epsilon$.

The function $f_{0}(x, u)$ is said to be seminormal in $u$ at the point $\bar{x} \in A$ if it has the just-mentioned property at $(\bar{x}, \bar{u}) \in A \times E_{m}$ for every $\bar{u} \in E_{m}$. These concepts of seminormality are essentially due to Tonelli (Ref. 13).

Requirement $\left(\mathrm{SN}^{\prime \prime}\right)$ is often stated in the stronger form $\left(\mathrm{SN}^{\prime *}\right)$ : $f_{0}(x, u) \leqslant r+b \cdot u+\epsilon$ for all $x \in N_{\delta}(\bar{x}), u \in E_{m},|u-\bar{u}| \leqslant \delta$. As we shall see, statement (6.i) below holds for both forms $\left(\mathrm{SN}^{\prime \prime}\right)$ and $\left(\mathrm{SN}^{\prime *}\right)$.

(6.i) If $f_{0}(x, u)$ is continuous in $A \times E_{m}$ and convex in $u$ for every $x \in A$, then $f_{0}$ is seminormal in $u$ at $\bar{x}$ if, and only if, for no $\bar{u}, u_{1} \in E_{m}, u_{1}=0$, it occurs that $f_{0}(\bar{x}, \bar{u})=2^{-1}\left[f_{0}\left(\bar{x}, \bar{u}+\lambda u_{1}\right)+f_{0}\left(\bar{x}, \bar{u}-\lambda u_{1}\right)\right]$ for all $\lambda \geqslant 0$.

This statement was proved by Tonelli (Ref. 13) under smoothness conditions on $F$. The proof below, based only on continuity and convexity properties, can be found in Turner (Ref. 16) and is repeated here for the convenience of the reader.

Proof. (a) Suppose $f_{0}(x, u)$ seminormal in $u$ at the point $\bar{x} \in A$. Then, for every $\vec{u} \in E_{m}$, there are constants $r, b=\left(b_{1}, \ldots, b_{m}\right)$ real and $\nu>0$ such that $\left(\mathrm{SN}^{\prime}\right)$ and $\left(\mathrm{SN}^{\prime \prime}\right)$ hold. Let $\zeta(u)$ denote $\zeta(u)=r+b \cdot u$. If $\sum_{j} \lambda_{j} u_{j}$ is any convex combination of points $u_{j} \in E_{m}$, with $\bar{u}=\sum_{j} \lambda_{j} u_{j}$, then

$$
f_{0}(\bar{x}, \bar{u}) \leqslant \zeta(\bar{u})+\epsilon=\sum_{j} \lambda_{j} \zeta\left(u_{j}\right)+\epsilon<\sum_{j} \lambda_{j} f_{0}\left(\bar{x}, u_{j}\right)+\epsilon,
$$

where $\epsilon$ is arbitrary. Thus, $f_{0}(\bar{x}, u)$ is convex in $u$ at the point $u=\vec{u}$. 
If there were points $\bar{u}, u_{1} \in E_{m}$ with $u_{1} \neq 0$ such that

$$
2^{-1}\left[f_{0}\left(\bar{x}, \bar{u}+\lambda u_{1}\right)+f_{0}\left(\bar{x}, \bar{u}-\lambda u_{1}\right)\right]=f_{0}(\bar{x}, \bar{u})
$$

for all real $\lambda$, then, by force of $\left(\mathrm{SN}^{\prime}\right)$,

$$
\begin{aligned}
f_{0}(\bar{x}, \bar{u}) & =2^{-1}\left[f_{0}\left(\bar{x}, \bar{u}+\lambda u_{1}\right)+f_{0}\left(\bar{x}, \bar{u}-\lambda u_{1}\right)\right] \\
& \geqslant 2^{-1}\left[\zeta\left(\bar{u}+\lambda u_{1}\right)+\zeta\left(\bar{u}-\lambda u_{1}\right)\right]+2 v|\lambda|\left|u_{1}\right|=\zeta(\bar{u})+2 v|\lambda|\left|u_{1}\right| .
\end{aligned}
$$

This is impossible since $\lambda$ can be arbitrarily large. We have proved the necessity of the condition.

(b) Let us assume that $f_{0}(x, u)$ is convex in $u$ and that, for no points $\bar{u}$, $u_{1} \in E_{m}, u_{1} \neq 0$, the relation $f_{0}(\bar{x}, \bar{u})=2^{-1}\left[f_{0}\left(\bar{x}, \bar{u}+\lambda u_{1}\right)+f_{0}\left(\bar{x}, \bar{u}-\lambda u_{1}\right)\right]$ holds for all $\lambda \geqslant 0$, and let us prove that $f_{0}$ is seminormal in $u$ at $\bar{x} \in A$. Let $\bar{u}$ be any point of $E_{m}$, and let $v(u)=r_{1}+b_{1} \cdot u$ be the supporting plane of $f_{0}(\tilde{x}, u)$ at $u=\bar{u}$. Let $w(u)=\bar{r}+\bar{b} \cdot u$ be the function satisfying the requirements of $(5 . \mathrm{x})$ for $f_{0}(\bar{x}, u)$ thought of as a function of $u$ alone. Then, for $0 \leqslant \alpha \leqslant 1$ and all $u$, we have

$f_{0}(\bar{x}, u)-[\alpha z v(u)+(1-\alpha) v(u)]=\alpha\left[f_{0}(\bar{x}, u)-v v(u)\right]+(1-\alpha)\left[f_{0}(\bar{x}, u)-v(u)\right] \geqslant 0$.

Let $\alpha_{0}, 0<\alpha_{0} \leqslant 1$, be so small that $\alpha_{0}|w(\bar{u})-v(\bar{u})|<\epsilon / 4$, and let

$$
z(u)=\alpha_{0} z(u)+\left(1-\alpha_{0}\right) v(u)-\epsilon / 4 .
$$

Then,

$$
\begin{aligned}
f_{0}(\bar{x}, u)-z(u) & =\alpha_{0}\left[f_{0}(\bar{x}, u)-w(u)\right]+\left(1-\alpha_{0}\right)\left[f_{0}(\bar{x}, u)-v(u)\right]+\epsilon / 4 \\
& \geqslant \epsilon / 4 \quad \text { for all } \quad u \in E_{n}, \\
\lim \left[f_{0}(\bar{x}, u)-z(u)\right] & =+\infty \quad \text { as }|u| \rightarrow+\infty \\
f_{0}(\bar{x}, \bar{u})-z(\bar{u}) & =v(\bar{u})-z(\bar{u})=\alpha_{0}[v(\bar{u})-v(\bar{u})]+\epsilon / 4 \leqslant \epsilon / 2 .
\end{aligned}
$$

From (9), we conclude that, for some $m>0$, we have

$$
\inf _{|u-\bar{u}|=m}\left[f_{0}(\bar{x}, u)-z(u)\right]>2 \epsilon .
$$

Now, define $\eta(x)=\inf \left[f_{0}(x, u)-z(u)\right]$, where inf is taken for $|u-\bar{u}|=m$. Then, $\eta(x)$ is a continuous function of $x$ for $x \in A$, and $\eta(\bar{x})>2 \epsilon$. 
Then, (8)-(9) above and the continuity of $\eta(x)$ imply that there is $\delta>0$ such that

$$
\begin{aligned}
f_{0}(x, u)-z(u) & >\epsilon / 8 \quad \text { for } \quad|x-\bar{x}| \leqslant \delta, \quad|u-\bar{u}| \leqslant m, \\
\eta(x) & >9 \epsilon / 8 \quad \text { for } \quad|x-\bar{x}| \leqslant \delta, \\
f_{0}(x, u) & <z(u)+\epsilon \quad \text { for }|x-\bar{x}| \leqslant \delta, \quad|u-\bar{u}| \leqslant \delta .
\end{aligned}
$$

Relation (13) is requirement ( $\left.\mathrm{SN}^{\prime \prime}\right)$ [actually, the stronger statement $\left(\mathrm{SN}^{\prime *}\right)$ ]. If $\nu=\epsilon / 8 m$, then (11) implies

$$
f_{0}(x, u)-z(u)-\nu|u-\bar{u}|>(\epsilon / 8)-\nu|u-\bar{u}|>\epsilon / 8-\epsilon / 8=0
$$

for $|x-\bar{x}| \leqslant \delta,|u-\bar{u}| \leqslant m$. For $|u-\bar{u}|>m$, let $\alpha=m /|u-\bar{u}|$, so $0<\alpha<1$, and let us define $u_{1}=\alpha(u-\bar{u})+\bar{u}$. Then, $\left|u_{1}-\bar{u}\right|=$ $(m /|u-\bar{u}|)|u-\bar{u}|=m, u_{1}=\alpha u+(1-\alpha) \bar{u}$, and thus, for $|x-\bar{x}| \leqslant \delta$,

$$
\begin{aligned}
f_{0}\left(x, u_{1}\right) & \leqslant \alpha f_{0}(x, u)+(1-\alpha) f_{0}(x, \bar{u}), \\
f_{0}\left(x, u_{1}\right)-z\left(u_{1}\right) & \leqslant \alpha\left[f_{0}(x, u)-z(u)\right]+(1-\alpha)\left[f_{0}(x, \bar{u})-z(\bar{u})\right], \\
f_{0}(x, u)-z(u) & \geqslant f_{0}(x, \bar{u})-z(\bar{u})+(1 / \alpha)\left\{\left[f_{0}\left(x, u_{1}\right)-z\left(u_{1}\right)\right]-\left[f_{0}(x, \bar{u})-z(\bar{u})\right]\right\} \\
& >0+(1 / \alpha)[\eta(x)-\epsilon]>(1 / \alpha)(9 \epsilon / 8-\epsilon)=\epsilon / 8 \alpha .
\end{aligned}
$$

Since $\alpha \nu|u-\bar{u}|=\epsilon / 8$, we have

$$
f_{0}(x, u)-z(u)-\nu|u-\bar{u}|>(\epsilon / 8 \alpha)-(\epsilon / 8 \alpha)=0,
$$

or $f_{0}(x, u)>z(u)+v|u-\bar{u}|$ for all $u$ and $|x-\bar{x}| \leqslant \delta$. This is requirement $\left(\mathrm{SN}^{\prime}\right)$. Statement $(6 . \mathrm{i})$ is thereby proved.

\section{A Third Criterion for Property $(Q)$}

We give here a simple criterion for property $(Q)$ of the sets $\tilde{Q}(x)$ of Section 3 for the case in which $f$ is linear in $u$.

(7.i) [A criterion for property $(Q)$ for $f$ linear in $u$.] If $A$ is closed, $U=E_{m}, M=A \times E_{m}$, if $f_{0}(x, u)$ is continuous on $M$, convex in $u$, and seminormal in $u$ at a point $\bar{x} \in A$, if $f(x, u)=B(x) u+C(x)$, where the matrices $B, C$ have entries continuous on $A$, then the sets $\widetilde{Q}(x)$ satisfy property $(Q)$ at $\bar{x}$. 
Proof. By seminormality, we know that there is a neighborhood $N_{\delta_{0}}(\bar{x})$ of $\bar{x}$ in $A$ and real numbers $r, b=\left(b_{1}, \ldots, b_{n}\right)$ such that $f_{0}(x, u)-r-b \cdot u \geqslant 0$ for all $x \in N_{\delta_{0}}(\bar{x}), u \in E_{m}$. By replacing $f_{0}$ by $f_{0}-r-b \cdot u$, if necessary, we see that it is not restrictive to assume $f_{0} \geqslant 0$ for all $x \in N_{\delta_{0}}(\bar{x})$ and $u \in E_{m}$.

Thus, $f_{0}(x, u) \geqslant 0$ for all $x \in N_{\delta_{0}}(\bar{x}), u \in E_{m}$, and the sets $\bar{Q}(x)$ are defined by $\left[\left(\varkappa^{0}, z\right) \mid z^{0} \geqslant f_{0}(x, u), z=f(x, u), u \in E_{m}\right]$. We have to prove that $\tilde{z}=\left(z^{0}, z\right) \in \bigcap_{0}$ cl co $\mathscr{Q}(\bar{x} ; \delta)$ implies $\tilde{z} \in \bar{Q}(\bar{x})$. Let $\tilde{z}$ be a given point $\tilde{z}=\left(z^{0}, \bar{z}\right) \in \bigcap_{\delta} \mathrm{cl}$ co $\mathscr{Q}(\bar{x} ; \delta)$, and let us prove that $\tilde{z} \in \tilde{Q}(\bar{x})$. For every $\delta>0$, we have $\bar{z}=\left(\bar{z}^{0}, \bar{z}\right) \in \mathrm{cl} \operatorname{co} \bar{Q}(\bar{x} ; \delta)$, and thus, for every $\delta>0$, there are points $z=\left(z^{0}, z\right) \in \operatorname{co} \bar{Q}(\tilde{x} ; \delta)$ at a distance as small as we want from $\tilde{z}=\left(\bar{z}^{0}, \bar{z}\right)$. Thus, there is a sequence of numbers $\delta_{k}>0$ and points $\tilde{z}_{k}=\left(z_{k}^{0}, z_{k}\right) \in \operatorname{co} \tilde{Q}\left(\bar{x} ; \delta_{k}\right)$ such that $\delta_{k} \rightarrow 0, \tilde{z}_{k} \rightarrow \tilde{z}$ as $k \rightarrow \infty$. In other words, for every integer $k$, there is a system of points $x_{k^{\gamma}} \in N_{\delta_{k}}(\bar{x}), \gamma=1, \ldots, \nu$, say $\nu=n+2$, corresponding points $\bar{z}_{k}{ }^{\gamma}=\left(z_{k}^{0 \gamma}, z_{k}^{\gamma}\right) \in \widetilde{Q}\left(x_{k}^{\gamma}\right)$, points $u_{k}{ }^{\gamma} \in E_{m}$, and numbers $\lambda_{k}{ }^{\gamma}, 0 \leqslant \lambda_{k}{ }^{\gamma} \leqslant 1, \gamma=1, \ldots, \nu$, such that

$$
\begin{aligned}
& 1=\sum \lambda_{k}{ }^{\gamma}, \quad \tilde{z}_{k}=\sum \lambda_{k}{ }^{\gamma} \tilde{z}_{k}{ }^{\gamma}, \quad z_{k}^{0}=\sum \lambda_{k}{ }^{\gamma} z_{k}^{0 \gamma}, \quad z_{k}=\sum \lambda_{k}{ }^{\gamma} z_{k}{ }^{\gamma}, \\
& z_{i}^{0 \gamma} \geqslant f_{0}\left(x_{k}^{\gamma}, u_{k}{ }^{\gamma}\right), \quad z_{k}^{\gamma}=f\left(x_{k}{ }^{\gamma}, u_{k}{ }^{\nu}\right)=B\left(x_{k}{ }^{\gamma}\right) u_{k}{ }^{\gamma}+C\left(x_{k}{ }^{\gamma}\right),
\end{aligned}
$$

where $\gamma=1, \ldots, v ; k=1,2, \ldots ;$ where $\sum$ ranges over all $\gamma=1, \ldots, v ; x_{k}{ }^{\gamma} \in N_{\delta_{k}}(\bar{x})$; and $x_{k}{ }^{\gamma} \rightarrow \bar{x}, z_{k_{b}} \rightarrow \overline{z_{1}} z_{l_{i}}{ }^{0} \rightarrow \bar{z}^{0}, z_{k} \rightarrow \bar{z}$ as $k \rightarrow \infty, \gamma=1, \ldots, \nu$.

By seminormality of $f_{0}$ at the point $\bar{x}$, there are numbers $\delta^{\prime}, 0<\delta^{\prime} \leqslant \delta_{0}$, $v>0, r$ real so that $f_{0}(x, u) \geqslant r+v|u|$ for all $x \in N_{\delta}(\bar{x})$. If $k$ is sufficiently large so that $\delta_{k}<\delta^{\prime} \leqslant \delta_{0}$, and hence $\left|x_{k}{ }^{\gamma}-\bar{x}\right| \leqslant \delta_{k}<\delta^{\gamma}$, and because $\zeta=r+\nu|u|$ is a convex function of $u$, we have

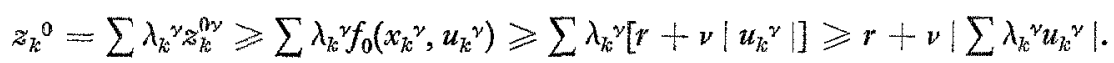

Thus, $\left|\sum \lambda_{k}{ }^{\gamma} u_{k}{ }^{\gamma}\right| \leqslant \nu^{-1}\left[z_{k}^{0}-r\right]$, where $z_{k}^{0} \rightarrow \bar{z}^{0}$ as $k \rightarrow \infty$. This proves that $\sum \lambda_{k}{ }^{y} u_{k}{ }^{v}, k=1,2, \ldots$, is a bounded sequence of points of $E_{m}$. By a suitable extraction, there is a subsequence, say still $[k]$, such that $u_{k}=\sum \lambda_{k}{ }^{\gamma} u_{k}{ }^{y} \rightarrow \vec{u} \in E_{m}$ as $k \rightarrow \infty$.

From the third of relations (14), where $\approx_{k}^{0} \rightarrow \bar{z}^{0}, z_{k}^{0_{\gamma}} \geqslant 0,0 \leqslant \lambda_{k^{y}} \leqslant 1$, we deduce that each of the $\nu$ sequences $\left[\lambda_{k}{ }^{\gamma} z_{k}^{0}, k=1,2, \ldots\right], \gamma=1, \ldots, \nu$, is bounded. From the fifth of relations (14), we then deduce that

$$
\lambda_{z^{\gamma}} z_{k}^{0 \gamma} \geqslant \lambda_{k}^{\gamma} f_{0}\left(x_{k}{ }^{\gamma}, u_{k}{ }^{\gamma}\right) \geqslant \lambda_{k}{ }^{\gamma}\left(r+\nu\left|u_{k}{ }^{\gamma}\right|\right),
$$

and hence $\lambda_{k}^{\gamma}\left|u_{k}^{\gamma}\right| \leqslant \nu^{-1}\left[\lambda_{k}^{\gamma} z_{k}^{\partial_{\gamma}}+|r|\right]$. Thus, each of the $\nu$ sequences $\left[\lambda_{k}{ }^{\gamma} u_{k}{ }^{\gamma}, k=1,2, \ldots,\right], \gamma=1,2, \ldots, \nu$, is bounded. 
If we denote by $\Delta_{k}^{\gamma}$ the expression

$$
\Delta_{k}{ }^{\gamma}=\lambda_{k}{ }^{\gamma}\left[\left(B\left(x_{k}{ }^{\gamma}\right) u_{k}{ }^{\gamma}+C\left(x_{k}{ }^{\gamma}\right)\right)-\left(B(\bar{x}) u_{k}{ }^{\gamma}+C(\bar{x})\right)\right],
$$

or

$$
\Delta_{k k^{y}}=\left(B\left(x_{k}{ }^{\gamma}\right)-B(\bar{x})\right)\left(\lambda_{k}{ }^{\gamma} u_{k}^{\gamma}\right)+\lambda_{k}^{\gamma}\left(C\left(x_{k}^{\gamma}\right)-C(\bar{x})\right),
$$

and because of the continuity of $B$ and $C$, and of $x_{k}{ }^{\gamma} \rightarrow \bar{x}, 0 \leqslant \lambda_{k}{ }^{\gamma} \leqslant 1$, we conclude that $\Delta_{k}{ }^{y} \rightarrow 0$ as $k \rightarrow \infty, \gamma=1, \ldots, \nu$.

Given $\epsilon>0$, by the seminormality of $f_{0}(x, u)$ at $\bar{x}$, we can determine new numbers $\delta^{\prime \prime}>0$ and $r, b=\left(b_{1}, \ldots, b_{n}\right)$ real so that

$$
\begin{aligned}
& f_{0}(x, u) \geqslant r+b \cdot u \quad \text { for all } x \in N_{\delta^{\prime \prime}}(\bar{x}), \quad u \in E_{m}, \\
& f_{0}(\bar{x}, \bar{u}) \leqslant r+b \cdot \bar{u}+\epsilon .
\end{aligned}
$$

Now, we have

$$
\begin{aligned}
z_{k}^{0} & =\sum \lambda_{k}{ }^{\gamma} z_{k}^{0 \gamma} \geqslant \sum \lambda_{k}{ }^{\gamma} f_{0}\left(x_{k}^{\gamma}, u_{k}{ }^{\gamma}\right) \geqslant \sum \lambda_{k}{ }^{\gamma}\left[r+b \cdot u_{k}{ }^{\gamma}\right]=r+b \cdot u_{k} \\
& =r+b \cdot \bar{u}+b \cdot\left(u_{k}-\bar{u}\right) \leqslant f_{0}(\bar{x}, \bar{u})+b \cdot\left(u_{k}-\bar{u}\right)-\epsilon, \\
z_{k} & =\sum \lambda_{k}{ }^{\gamma} z_{k}{ }^{\gamma}=\sum \lambda_{k}\left[B\left(x_{k}\right) u_{k}{ }^{\gamma}+C\left(x_{k}\right)\right]=\sum \lambda_{k}\left[B(\bar{x}) u_{k}{ }^{\gamma}+C(\bar{x})\right]+\Delta_{k} \\
& =B(\bar{x}) u_{k}+C(\bar{x})+\Delta_{k} .
\end{aligned}
$$

At the limit, as $k \rightarrow \infty$, we obtain

$$
\bar{z}^{0} \geqslant f_{0}(\bar{x}, \bar{u})-\epsilon, \quad \bar{z}=B(\bar{x}) \bar{u}+C(\bar{x}),
$$

and, because $\epsilon>0$ is arbitrary, also $\bar{z}^{0} \geqslant f_{0}(\bar{x}, \bar{u}), \bar{z}=f(\bar{x}, \bar{u})$; hence, $\tilde{z}=\left(\bar{z}^{0}, \bar{z}\right) \in \widetilde{Q}(\bar{x})$. Statement $(7 . \mathrm{i})$ is thereby proved.

\section{The Function $T$}

Given $A, U(x), M, f_{0}, f=\left(f_{1}, \ldots, f_{n}\right), Q(x), \Phi(x)$ as usual $(A, M$ closed, $f_{0}, f$ continuous on $\left.M\right)$, let us recall here that the sets $Q(x)$ are the projections of the sets $\Phi(x) \subset E_{n+1}$ on the z-space $E_{n}$. Hence, for every $\left(z^{0}, z\right) \in \widetilde{Q}(x)$, we have $z \in Q(x)$, and $z=f(x, u), z^{0} \geqslant f_{0}(x, u)$ for some $u \in U(x)$. Conversely, if $z \in Q(x)$, hence $z=f(x, u)$ for some $u \in U(x)$, all points $\left(z^{0}, z\right)$ with $z^{0} \geqslant f_{0}(x, u)$ certainly are in $\mathscr{Q}(x)$.

For any fixed $\bar{x} \in A$, let us consider the following scalar function defined on $\bar{Q}(\bar{x})$ :

$$
\begin{aligned}
T(z ; \bar{x}) & =\inf \left[f_{0}(\bar{x}, u) \mid z=f(\bar{x}, u), \quad u \in U(\bar{x})\right] \\
& =\inf \left[z^{0} \mid\left(z^{0}, z\right) \in \bar{Q}(\bar{x})\right], \quad z \in Q(\bar{x}) .
\end{aligned}
$$


Then, for $\bar{x} \in A$, we have $-\infty \leqslant T(z ; \bar{x})<+\infty$ for all $z \in Q(\bar{x})$. We shall consider $T(z ; \bar{x})$ as a function of $z$ in $Q(\bar{x})$.

Note that the convexity of $\widetilde{Q}(\bar{x}) \subset E_{n+1}$ implies the convexity of $Q(\bar{x}) \subset E_{n}$, but $Q(\bar{x})$ may not be closed even if $\Phi(\bar{x})$ is closed. Also, we shall denote by $R$ the linear manifold in $E_{n}$ containing $Q(\bar{x})$ of minimum dimension $r$; thus, $Q(\bar{x}) \subset R \subset E_{n}, 0 \leqslant r \leqslant n$. As usual, we shall denote by int $Q(\bar{x})$ the set of all $z \in E_{n}$ which are interior to $Q(\bar{x})$ with respect to $E_{n}$ and by $\operatorname{Rint} Q(\bar{x})$ the set of all points $z$ which are interior to $Q(\bar{x})$ with respect to $R$; thus, int $Q(\bar{x}) \subset \operatorname{Rint} Q(\bar{x}) \subset Q(\bar{x}) \subset R \subset E_{n}$.

(8.i) If $\bar{Q}(\bar{x})$ is convex, then either $T(z ; \bar{x})=-\infty$ for all $z \in \operatorname{Rint} Q(\bar{x})$ or $T(z ; \bar{x})>-\infty$ for all $z \in Q(\bar{x})$. In the latter case, $T(z, \bar{x})$ is finite everywhere and a convex function of $z$ in $Q(\bar{x}), T(z ; \bar{x})$ is bounded below on every bounded subset of $Q(\bar{x})$, and $T(z ; \bar{x})$ is continuous in the convex set Rint $Q(\bar{x})$ open with respect to $R$. Finally, if $\tilde{Q}(\bar{x})$ is convex and closed, and $T(z, \bar{x})>-\infty$ for all $z \in Q(\bar{x})$, then $T(z ; \bar{x})$ is lower semicontinuous at every point $z \in Q(\bar{x})-\operatorname{Rint} Q(\bar{x})$.

Proof. If $Q(\bar{x})$ is a single point, then $r=0, \operatorname{Rint} Q(\bar{x})=\varnothing$, and nothing has to be proved. Assume that $Q(\bar{x})$ is not a single point. Then, $1 \leqslant r \leqslant n$, and $\operatorname{Rint} Q(\bar{x}) \neq \varnothing$. Let $\bar{z}$ be any point $\bar{s} \in \operatorname{Rint} Q(\bar{x})$. Assume that, at some point $z_{1} \in Q(\bar{x})$, we have $T\left(z_{1}, \bar{x}\right)=-\infty$, and let us prove that $T(\bar{z} ; \bar{x})=-\infty$. For any integer $k$, there are points $\left(z_{k}^{0}, z_{1}\right) \in \widetilde{Q}(\bar{x})$ with $z_{k}{ }^{0}<-k, k=1,2, \ldots$. Take $\lambda=z_{1}-\bar{z}$, and choose $\delta>0$ so small that $z_{2}=\bar{z}-\lambda \delta \in \operatorname{Rint} Q(\bar{x})$. Take any point $\left(z_{2}{ }^{0}, z_{2}\right) \in \bar{Q}(\bar{x})$ and note that all points

$$
\left(\alpha z_{2}^{0}+(1-\alpha) z_{k}^{0}, \alpha z_{2}+(1-\alpha) z_{1}\right), \quad 0 \leqslant \alpha \leqslant 1,
$$

belong to $\Phi(\bar{x})$. In particular, for $\alpha=(1+\delta)^{-1}$, we have

$$
\begin{aligned}
\alpha z_{2}+(1-\alpha) z_{1} & =\alpha(\bar{z}-\lambda \delta)+(1-\alpha) z_{1} \\
& =\bar{z}-(1-\alpha)\left(\bar{z}-z_{1}\right)-\alpha \lambda \hat{\delta}=\bar{z}+\lambda(1-\alpha-\alpha \delta)=\bar{z}, \\
T(\bar{z} ; \bar{x}) & \leqslant \alpha z_{2}^{0}+(1-\alpha) z_{k}^{0} \leqslant(1+\delta)^{-1} z_{2}{ }^{0}-\left(1-(1+\delta)^{-1}\right) k,
\end{aligned}
$$

where the last term approaches $-\infty$ as $k \rightarrow \infty$; hence, $T(\bar{z} ; \bar{x})=-\infty$. Since $\bar{x}$ is any point of $\operatorname{Rint} Q(\bar{x})$, we have proved the first part of $(8 . i)$.

The remaining parts of (8.i) are now a consequence of the definitions and statements (5.v), (5.vi), (5.viii).

The first of the two cases mentioned in (8.i) may actually occur, even in situations where the sets $\tilde{Q}$ have property $(Q)$ at $\bar{x}$. Indeed, take $m=n=1$, 
$f_{0}=u, f=0, U=E_{1}$. Then, $Q=[z \mid z=0], \tilde{Q}=\left[\left(z^{0}, z\right) \mid z^{0} \in E_{1}, z=0\right]$, and $T=-\infty$. As another case, take $n=1, m=2, u, v$ control variables, $f_{0}=u, f=\sin v, U=\left[(u, v) \in E_{2}\right]$. Then, $Q=[z \mid-1 \leqslant z \leqslant 1]$, $\widetilde{Q}=\left[\left(z^{0}, z\right) \mid z^{0} \in E_{1},-1 \leqslant z \leqslant 1\right]$, and $T(z)=-\infty$ for all $-1 \leqslant z \leqslant 1$. In both cases, $Q$ and $\Phi$ are fixed, closed, convex sets, and certainly have property $(Q)$. As a third example, take $n=1, m=2, u$, $v$ control variables, $f_{0}=\left(1-\sin ^{2} v\right) u, f=\sin v, U=\left[(u, v) \in E_{2}\right]$. Then, $Q=[z \mid-1 \leqslant z \leqslant 1]$, and $\bar{Q}=\left[\left(z^{0}, z\right) \mid z^{0} \in E_{1}\right.$ if $-1<z<1$, and $z^{0}=0$ if $\left.z= \pm 1\right]$. Finally, $T(z)=-\infty$ for $-1<z<1, T(z)=0$ for $z= \pm 1$.

The following example proves that $T(z ; \bar{x})$ may not be lower semicontinuous on $Q(\bar{x})$ if the set $\widetilde{Q}(\bar{x})$ is not closed. As usual, we shall denote by $[g(P)]_{h}$ the function of $P$ which has the value $g(P)$ if $g(P) \geqslant h$ and the value $h$ if $g(P)<h$. Now, take $n=1, m=2, u$, $v$ control variables, $f_{0}=\left[\left(1-\sin ^{2} v\right) u\right]_{-1}, f=\sin v, U=\left[(u, v) \in E_{2}\right]$. Then, $Q=[z \mid-1 \leqslant z \leqslant 1]$, and $\bar{Q}=\left[\left(z^{0}, z\right) \mid z^{0} \geqslant-1\right.$ if $-1<z<1, z^{0} \geqslant 0$ if $\left.z= \pm 1\right]$. Finally, $T(z)=-1$ for $-1<z<1, T(z)=0$ for $z= \pm 1$.

The following example shows that, even if the set $\widetilde{Q}(\bar{x})$ is closed and convex, the function $T(z ; \bar{x})$ may not be continuous at the points $z \in Q(\bar{x})-R$ int $Q(\bar{x})$. Let $Q$ be the convex set $\left[(\xi, \eta) \mid 0 \leqslant \xi \leqslant 1,0 \leqslant \eta \leqslant \sqrt{ }\left(1-(1-\xi)^{2}\right)\right]$, and let $T(\xi, \eta)$ be defined by taking $T=\eta$ for $0 \leqslant \xi \leqslant 1,0 \leqslant \eta \leqslant \xi$, $T=(2 \xi)^{-1}\left(\xi^{2}+\eta^{2}\right)$ for $0<\xi<1, \xi \leqslant \eta \leqslant \sqrt{ }\left(1-(1-\xi)^{2}\right)$. As we have seen in Section 5, $T(\xi, \eta)$ is convex and bounded in $Q$ and continuous in $Q$ except at the point $(\xi=0, \eta=0)$. Now let us define the set $\tilde{Q}$. To this purpose, let $U$ be the union of the two closed disjoint sets

$$
U_{1}=[(u, v, w) \mid 0 \leqslant u \leqslant 1,-1 \leqslant v \leqslant u-1, w \geqslant 0]
$$

and

$$
U_{2}=\left[(u, v, w) \mid 0 \leqslant u \leqslant 1, u \leqslant v \leqslant \sqrt{ }\left[1-(1-u)^{2}\right], w \geqslant 0\right] .
$$

Let $\sigma(w)=(w+1)^{-1}, w \geqslant 0$. Finally, let us define the functions $f_{0}(u, v, w)$, $f_{1}(u, v, w), f_{2}(u, v, w)$, continuous on $U=U_{1} \cup U_{2}$, by taking $f_{1}=u, f_{2}=v+1$, $f_{0}=v$ on $U_{1}$, and

$$
\begin{aligned}
& \xi=f_{1}=u, \\
& \eta=f_{2}=v, \\
& f_{0}=\left[\sigma(w)+(1-\sigma(w))\left(u^{2}+v^{2}\right)\right] /[\sigma(w)+2(1-\sigma(w)) u]
\end{aligned}
$$


on $U_{2}$. Then, if $Q$ denotes the set

$$
\check{Q}=\left[\left(z^{0}, \xi, \eta\right) \mid z^{0} \geqslant f_{0}, \xi=f_{1}, \eta=f_{2},(u, v, w) \in U=U_{1} \cup U_{2}\right],
$$

and

$$
T(\xi, \eta)=\inf \left[z^{0} \mid\left(z^{0}, \xi, \eta\right) \in \widetilde{Q}\right],
$$

then $T$ is exactly the convex function defined above on $Q$, and $\widetilde{Q}$ is convex and closed.

The following example shows that, at points $\bar{z} \in Q(\bar{x})-\operatorname{Rint} Q(\bar{x})$, the supporting plane of $\widetilde{Q}(\bar{x})$ may be vertical even if $\tilde{Q}(\bar{x})$ is convex and closed, $Q(\bar{x})$ is convex and compact, and $T(z ; \bar{x})$ continuous on $Q(\bar{x})$. Indeed, take

$Q=\left[(u, v) \mid u^{2}+v^{2} \leqslant 1\right], T=-\sqrt{ }\left(1-u^{2}-v^{2}\right), U=Q, f_{1}=u, f_{2}=v, f_{0}=T$, $\widetilde{Q}=\left[\left(z^{0}, u, v\right) \mid z^{0} \geqslant T,(u, v) \in U\right]$.

\section{A Characterization of Property $(Q)$ for the Sets $\underline{Q}(t, x)$}

For fixed $\bar{x} \in A$ and $\delta>0$, let us consider the set

$$
\widetilde{Q}^{*}(\bar{x} ; \delta)=\operatorname{co} \widetilde{Q}(\widetilde{x} ; \delta)=\operatorname{co}\left\{\bigcup_{x \in N_{\delta}(\tilde{x})} \widetilde{Q}(x)\right\} \subset E_{n+1}
$$

and its projection on the $z$-space $E_{n}$

$$
Q^{*}(\bar{x} ; \delta)=\operatorname{co} Q(\bar{x} ; \delta)=\operatorname{co}\left\{\bigcup_{x \in N_{\delta}(\tilde{x})} Q(x)\right\} \subset E_{n} .
$$

Both sets $\tilde{Q}^{*}(\bar{x} ; \delta)$ and $Q^{*}(\bar{x} ; \delta)$ are convex, and

$$
E_{n+1} \supset \bar{Q}(\bar{x} ; \delta) \supset \tilde{Q}(\bar{x}), \quad E_{n} \supset Q^{*}(\bar{x} ; \delta) \supset Q(\bar{x}) .
$$

As before, we shall consider the following scalar function defined on $Q^{*}(\bar{x} ; \delta):$

$$
T^{*}(z ; \bar{x}, \delta)=\inf \left\{z^{0} \mid\left(z^{0}, z\right) \in \widetilde{Q}^{*}(\bar{x} ; \delta)\right\}, \quad z \in Q^{*}(\bar{x} ; \delta) .
$$

Thus, we have again $-\infty \leqslant T^{*}(z ; \bar{x}, \delta)<\infty$ for all $z \in Q^{*}(\bar{x} ; \delta)$. Also, for every $z \in Q(\bar{x})$, we have $T^{*}(z ; \bar{x}, \delta) \leqslant T(z ; \bar{x})$. We now have the following characterization of property $(Q)$ : 
(9.i) If $T(z ; \bar{x})>-\infty$ in $Q(\bar{x})$, then the sets $\tilde{Q}(x)$ have property $(Q)$ at $\bar{x}$ if, and only if, properties $(\alpha)$ and $(X)$ hold at the point $\bar{x}$.

Proof. We have already proved in (4.i) that the union of $(\alpha)$ and $(X)$ implies $(Q)$. We need only prove that, if $T(z ; \bar{x})>-\infty$ in $Q(\bar{x})$ and $\mathscr{Q}(x)$ has property $(Q)$ at $\bar{x}$, then both $(\alpha)$ and $(X)$ hold at $\bar{x}$. We know already that $(\alpha)$ is a necessary condition for property $(Q)$, and thus $(\alpha)$ holds. Also, $\bar{Q}(\bar{x})$ is closed and convex. Since $T(z ; \bar{x})>-\infty$ by hypothesis, we know from (8.i) that $T(z ; \bar{x})$ is a lower-semicontinuous convex function of $z$ in the convex set $Q(\bar{x})$. We have already noticed that $-\infty \leqslant T^{*}(z ; \bar{x}, \delta) \leqslant T(z, \bar{x})<+\infty$ for all $z \in Q(\bar{x})$ and $\delta>0$.

Now, take any point $\bar{z} \in Q(\bar{x})$, and let $\bar{z}^{0}=T(\bar{z}, \bar{x})$. Then, by (8.i), the point $\left(\bar{z}^{0}, \bar{z}\right)$ belongs to $\tilde{Q}(\bar{x})$, and hence there is some $\bar{u} \in U(\bar{x})$ with $\bar{z}^{0}=T(\bar{z}, \bar{x})=f_{0}(\bar{x}, \bar{u}), \bar{z}=f(\bar{x}, \bar{u})$. Given $\epsilon>0$, the point $\bar{P}=\left(\bar{z}^{0}-\epsilon, \bar{z}\right)$ is not on the closed set $\bar{Q}(\bar{x})$, and hence has a minimum distance $\eta$ from this set, with $0<\eta \leqslant \epsilon$. Since $T(z ; \bar{x})$ is lower semicontinuous at $\bar{z}$, there is some $\eta^{\prime}, 0<\eta^{\prime} \leqslant \eta$, such that $T(z ; \bar{x})>T(\bar{z} ; \bar{x})-\eta / 3$ for all $z \in Q(\bar{x})$ with $|z-\bar{z}| \leqslant \eta^{\prime}$.

Let $\sigma$ be the closed ball in $E_{n+1}$ of center $\bar{P}=\left(\bar{z}^{0}-\epsilon, \bar{z}\right)$ and radius $\eta^{\prime} / 3$. Let $\sigma_{0}$ denote the projection of $\sigma$ on the $z$-space; thus, $\sigma_{0}$ is the closed ball in $E_{n}$ of center $\bar{z}$ and radius $\eta^{\prime} / 3$. We shall denote also by $\sigma_{1}$ the closed ball in $E_{n}$ of center $\bar{z}$ and radius $2 \eta^{\prime} / 3$.

Now let us consider the convex sets $\bar{Q}^{*}(\bar{x} ; \delta)=$ co $\bar{Q}(\bar{x} ; \delta)$ defined in $(15)$ and their relative functions $T^{*}(z ; \bar{x}, \delta)$ defined in (16). Let us prove that there is some $\delta_{0}>0$ such that

$$
0 \leqslant T(\bar{z} ; \bar{x})-T^{*}(z ; \bar{x}, \delta)<\eta / 3
$$

for all $0<\delta \leqslant \delta_{0}$ and $z \in \sigma_{1} \cap Q^{*}(\bar{x} ; \delta)$. Indeed, in the contrary case, there would be numbers $\delta_{k}>0$ and points $z_{k} \in \sigma_{1} \subset E_{n}, k=1,2, \ldots$, with $\delta_{k} \rightarrow 0$ as $k \rightarrow \infty$ and $T^{*}\left(z_{k} ; \bar{x}, \delta_{k}\right) \leqslant T(\bar{z}, \bar{x})-\eta / 3$, and hence points $\left(z_{k}^{0}, z_{k}\right) \in \operatorname{co} \bar{Q}\left(\bar{x}, \delta_{k}\right)$ with $z_{k}{ }^{0} \leqslant T(\bar{z} ; \bar{x})-\eta / 3=\bar{z}^{0}-\eta / 3$. Hence, for every $\delta>0$, we have $\left(z_{k}^{0}, z_{k}\right) \in \operatorname{co} \bar{Q}(\bar{x} ; \delta)$ for all $k$ sufficiently large, and then also $\left(\bar{z}^{0}-\eta / 3, z_{k}\right) \in \operatorname{co} \widetilde{Q}(\bar{x} ; \delta)$. If $\bar{z}^{\prime}$ is any point of accumulation of $\left[z_{k}\right]$, we have then $\bar{z}^{\prime} \in \sigma_{1}, \quad\left(\bar{z}^{0}-\eta / 3, \bar{z}^{\prime}\right) \in \mathrm{cl} \operatorname{co} \bar{Q}(\bar{x} ; \delta)$, and by property $(Q)$ also $\left(\bar{z}^{0}-\eta / 3, \bar{z}^{\prime}\right) \in \widetilde{Q}(\bar{x})=\bigcap_{\delta}$ cl co $\widetilde{Q}(\bar{x} ; \delta)$. This implies $T\left(\bar{z}^{\prime} ; \bar{x}\right) \leqslant \bar{z}^{0}-\eta / 3$ with $\bar{z}^{\prime} \in \sigma_{1},\left|\bar{z}^{\prime}-\bar{z}\right| \leqslant 2 \eta^{\prime} / 3<\eta^{\prime}$, a contradiction. We have proved that, for some $\delta_{0}>0$, relation (17) holds for all $0<\delta \leqslant \delta_{0}$ and $z \in \sigma_{1} \cap Q^{*}(\bar{x}, \delta)$.

Let us prove that any two points

$$
P=\left(z^{0}, z\right) \in \sigma \quad \text { and } \quad P^{\prime}=\left(z^{\prime 0}, z^{\prime}\right) \in \mathscr{Q}^{*}\left(\bar{x} ; \delta_{0}\right)
$$


have a distance $\left\{P, P^{\prime}\right\} \geqslant \eta^{\prime} / 3$. Indeed, either $P^{\prime}$ is outside the cylinder $\left(z^{0} \in E_{1}, z \in \sigma_{1}\right)$, and then

$$
\left\{P^{\prime}, P\right\} \geqslant\left|z^{\prime}-z\right| \geqslant\left|z^{\prime}-\bar{z}\right|-|z-\bar{z}| \geqslant 2 \eta^{\prime} / 3-\eta^{\prime} / 3=\eta^{\prime} / 3,
$$

or $P^{\prime}$ is inside the cylinder above, and then, by (17), for $0<\delta \leqslant \delta_{0}$,

$$
\begin{aligned}
z^{\prime 0} & \geqslant T^{*}\left(z^{\prime} ; \bar{x}, \delta_{0}\right)>T(\bar{z}, \bar{x})-\eta / 3=\bar{z}^{0}-\eta / 3, \\
\left\{P^{\prime}, P\right\} & \geqslant z^{\prime 0}-z^{0}=\left[\bar{z}^{0}-\left(\bar{z}^{0}-\epsilon\right)\right]+\left[z^{\prime 0}-\bar{z}^{0}\right]+\left[\bar{s}^{0}-\epsilon-z^{0}\right] \\
& \geqslant \epsilon-\eta / 3-\{\bar{P}, P\} \geqslant \eta-\eta / 3-\eta / 3=\eta / 3 .
\end{aligned}
$$

Thus, the convex sets $\sigma$ and $\tilde{Q}^{*}(\bar{x} ; \delta)$ have a distance $\geqslant \eta / 3$, and the same occurs for the convex closed sets $\sigma$ and $\operatorname{cl} \tilde{Q}^{*}(\bar{x}, \delta)$, o compact. We conclude that there is some hyperplane $\Pi$ in $E_{n+1}$ separating the two convex sets $\sigma$ and $\mathrm{dl} \bar{Q}^{*}(\bar{x} ; \delta)$.

This hyperplane $\Pi$ must intersect the vertical segment

$$
\left[\bar{z}^{0}-\epsilon+\eta / 3 \leqslant z^{0} \leqslant \bar{z}^{0}, z=\bar{z}\right]
$$

at some point $\left(z^{0}=r, z=\bar{z}\right)$, and $\Pi$ cannot be parallel to the $z^{0}$-axis, otherwise all points of the straight line $z=\bar{z}$ would be on $I$, in particular, the center $\bar{P}$ of the ball $\sigma$, and not all points of $\sigma$ could be separated from $\bar{Q}^{*}(\bar{x} ; \delta)$. Thus, $\Pi$ is of the form

$$
\Pi: \quad z=r+b \cdot(z-\bar{z})=(r-b \cdot \bar{z})+b \cdot z,
$$

$\bar{Q}(\bar{x})$ as well as $\mathrm{cl} \bar{Q}^{*}(\bar{x} ; \delta)$ are above $\Pi$, and thus $\left(z^{0}, z\right) \in \operatorname{cl} \tilde{Q}^{*}(x ; \delta)$ implies $z^{0} \geqslant(r-b \cdot \bar{z})+b \cdot z$. In other words, for $0<\delta \leqslant \delta_{0}, x \in N_{\delta}(\bar{x}), x \in A$, $u \in U(x)$, we have $f_{0}(x, u) \geqslant(r-b \cdot \bar{z})+b \cdot f(x, u)$. On the other hand, $f_{0}(\bar{x}, \bar{u})=\bar{z}^{0}=\left(\bar{z}^{0}-\epsilon\right)+\epsilon<r+\epsilon=(r-b \cdot \bar{z})+b \cdot \bar{z}+\epsilon$. We have proved that property $(X)$ holds at the point $\bar{x} \in A$. Statement (9.i) is thereby proved.

We now state, for the convenience of the reader, a criterion for property $(Q)$ recently proved by Olech (Ref. 6). Let us consider again the subsets $Q(x)$ defined in Section 3 of the $\tilde{z}$-space $E_{n+1}, \tilde{z}=\left(z^{0}, z\right)=\left(z^{0}, z^{1}, \ldots, z^{n}\right)$. We shall denote by $\tilde{c}=\left(c^{0}, c\right)=\left(c^{0}, c^{1}, \ldots, c^{n}\right), \tilde{d}=\left(d^{0}, d\right)$, $\tilde{z}=\left(z^{0}, z\right)$ arbitrary points in $E_{n+1}$. For any point $x \in A$, let $C(x)$ denote the set

$$
C(x)=\left[\tilde{c} \in E_{n+\mathbf{1}} \mid \tilde{z}+\lambda \tilde{c} \in \tilde{Q}(x) \text { for all } \lambda \geqslant 0 \text { and } \tilde{z} \in \tilde{Q}(x)\right] \text {. }
$$

Obviously, $C(x)$ is a cone of vertex $\tilde{c}=0$ in $E_{n+1}$. The set $C(x)$ is said to be the asymptotic cone of the set $\Phi(x)$. It is easy to see that, if $\mathscr{Q}(x)$ is convex, so 
is $C(x)$; if $\Phi(x)$ is closed, so is $C(x)$. Let $\Gamma$ denote the particular cone in $E_{n+1}$ made up of the only positive half axis $z^{0} \geqslant 0$, or

$$
\Gamma=\left[\tilde{z}=\left(z^{0}, z\right) \mid z^{0} \geqslant 0, z=0\right] .
$$

Then, $\Gamma \subset C(x)$ for all $x \in A$.

We shall denote by $C^{0}(x)$, or polar cone of $C(x)$, the set

$$
C^{0}(x)=\left[\tilde{d} \in E_{n+1} \mid \tilde{d} \cdot \tilde{c} \leqslant 0 \text { for all } \tilde{c} \in C(x)\right] .
$$

If $C(x)=\Gamma$, then obviously $C^{0}=\Gamma^{0}=\left[\tilde{d}=\left(d^{0}, d\right) \in E_{n+1} \mid d^{0} \leqslant 0, d \in E_{n}\right]$.

(9.ii) [A criterion for property $(Q)$.$] If the sets Q(x)$ satisfy property $(U)$ in $A$ (hence, they are closed and their graph is closed), if they are convex and do not contain any line, if the asymptotic cone is constant, or $C(x)=C=$ const, and for each number $r>0$ and point $\tilde{d} \in$ int $C^{0}, C^{0}$ the polar cone of $C$, we have

$$
\sup _{x \in A,|x| \leqslant r \mid \leqslant r} \sup _{\tilde{z} \in \tilde{Q}(x)}(\tilde{d} \cdot \tilde{z})<+\infty,
$$

then the sets $\widetilde{Q}(x)$ satisfy property $(Q)$ in $A$ (see Olech, Ref. 6, p. 169).

\section{References}

1. Kuratowski, C., Les Fonctions Semicontinues dans l'Espace des Ensembles Fermés, Fundamenta Mathematicae, Vol. 18, 1932.

2. Cesari, L., Existence Theorems for Weak and Usual Optimal Solutions in Lagrange Problems with Unilateral Constraints, I and II, Transactions of the American Mathematical Society, Vol. 124, No. 3, 1966.

3. Cesari, L., Existence Theorems for Optimal Controls of the Mayer Type, SIAM Journal on Control, Vol. 6, No. 4, 1968.

4. La Palm, J. R., Remarks on Certain Growth Conditions in Problems of Optimal Control, Journal of Optimization Theory and Applications, Vol. 4, No. 5, 1969.

5. Lasota, A., and Olfch, C., On the Closedness of the Set of Trajectories of a Control System, Bulletin de l'Académie Polonaise des Sciences, Série des Sciences Mathématiques, Astronomiques et Physiques, Vol. 14, 1966.

6. Olech, C., Existence Theorems for Optimal Problems with Vector-Valued Cost Functions, Transactions of the American Mathematical Society, Vol. 136, 1969.

7. Olecr, C., Existence Theorems for Optimal Control Problems Involving Multiple Integrals, Journal of Differential Equations, Vol. 6, 1969. 
8. Cesari, L., La Palm, J. R., and Nishiura, T., Remarks on Some Existence Theorems for Optimal Control, Journal of Optimization Theory and Applications, Vol. 3, No. 5, 1969.

9. Cesari, L., Existence Theorems for Multidimensional Problems of Optimal Control, Differential Equations and Dynamical Systems, Edited by J. K. Hale and J. P. Lasalle, Academic Press, New York, 1967.

10. Cesari, L., Existence Theorems for Multidimensional Lagrange Problems, Journal of Optimization Theory and Applications, Vol. 1, No. 2, 1967.

11. Cesari, L., Sobolev Spaces and Multidimensional Lagyange Problems of Optimization, Annali della Scuola Normale Superiore di Pisa, Vol, 22, No. 3, 1968.

12. Cesari, L., Existence Theorems for Abstract Multidimensional Control Problems, Journal of Optimization Theory and Applications, Vol. 6, No. 3, 1970.

13. Tonelli, L., Sugli Integrali del Calcolo delle Variazioni in Forma Ordinaria, Annali della Scuola Normale Superiore di Pisa, Vol. 3, No. 2, 1934 (see also Opere Scelte, Vol. 3, Edizioni Cremonese, Roma, Italy, 1962).

14. Choquet, G., Convergences, Annales de l'Université de Grenoble, Vol. 23, $1947-48$.

15. Michael, E., Topologies on the Spaces of Subsets, Transactions of the American Mathematical Society, Vol. 71, No. 1, 1951.

16. TuRner, L. H., The Direct Method in the Calculus of Variations, Purdue University, Ph.D. Thesis, 1957. 